\title{
An electrophysiological study of response conflict processing across the lifespan: Assessing the roles of conflict monitoring, cue utilization, response anticipation, and response suppression
}

\author{
Dorothea Hämmerer*, Shu-Chen Li, Viktor Müller, Ulman Lindenberger \\ Center for Lifespan Psychology, Max Planck Institute for Human Development, Lentzeallee 94, 14195 Berlin, Germany
}

\section{A R T I C L E I N F O}

\section{Article history:}

Received 17 August 2009

Received in revised form 11 June 2010

Accepted 9 July 2010

Available online 16 July 2010

\section{Keywords:}

Lifespan development

Response inhibition

Motor control

Cognitive control

Attention

ERP

\begin{abstract}
A B S T R A C T
We recorded Electroencephalograms (EEGs) during a cued Continuous Performance Task (CPT) to investigate lifespan differences in the efficiency of response conflict processing under conditions that put high demands on the ability to suppress a prepotent response. Previous evidence indicates that children and adolescents commit more errors under such conditions than younger adults, whereas older adults are disproportionately slow in responding. We measured event-related potentials (ERPs) in a sample of 45 children, 44 adolescents, 46 younger adults, and 47 older adults to investigate response conflict monitoring (Nogo-N2), cue utilization (Cue-P3), response anticipation (contingent negative variation, CNV), and response suppression (Nogo-P3). In comparison to adolescents and adults, children showed larger ERPs associated with cue utilization. At the same time, children committed more errors and their ERPs reflecting response anticipation and response suppression were smaller and uncorrelated. In contrast, older adults showed ERP indices of attentional distraction (P3a elicited by the infrequent Non-Cue stimuli), reduced conflict monitoring signals (Nogo-N2), and took more time to respond than the other age groups. The present findings reveal marked lifespan differences in processes related to response conflict monitoring. In middle childhood, the readiness to utilize cues for guiding actions is not yet fully matched by the ability to suppress prepotent responses, leading to a relatively large number of commission errors. In older adults, higher indices of attentional distraction as well as lower conflict monitoring signals were observed. This might reflect a dampened build-up of response tendencies, thereby leading to slower responding and relatively low error rates.
\end{abstract}

(c) 2010 Elsevier Ltd. All rights reserved.

\section{Introduction}

The last decade has seen a rapidly growing interest in studying conflict monitoring. Conflict monitoring is thought to come into play in challenging and non-routine situations that involve several concurring and potentially conflicting response options (see Botvinick, Braver, Barch, Carter, \& Cohen, 2001; Botvinick, Cohen, \& Carter, 2004; Ridderinkhof, Ullsperger, Crone, \& Nieuwenhuis, 2004; Rushworth, Walton, Kennerley, \& Bannerman, 2004; Yeung, Botvinick, \& Cohen, 2004 for reviews). For example, conflict monitoring has been suggested to take place during tasks involving response suppression, signaling the need to detect and control the conflict between the actions of executing or suppressing a response (Braver, Barch, Gray, Molfese, \& Snyder, 2001; Nieuwenhuis, Yeung, van den Wildenberg, \& Ridderinkhof, 2003).

\footnotetext{
* Corresponding author. Tel.: +49 3082406 463; fax: +49 308249939.

E-mail address: haemmerer@mpib-berlin.mpg.de (D. Hämmerer).
}

\subsection{Lifespan differences in conflict monitoring and associated processes}

The ability to process response conflicts differs across the human lifespan, with children and older adults being less efficient than younger adults. Moreover, when comparing the performance during maturation and aging, a distinct pattern of difficulties in processing response conflicts emerges. While children and adolescents are more likely to commit errors in the context of response conflicts than young adults (e.g., Davies, Segalowitz, \& Gavin, 2004; Jonkman, 2006; Jonkman, Lansbergen, \& Stauder, 2003; Lorsbach \& Reimer, 2008), difficulties in dealing with response conflicts are evident in disproportionately slowed response latencies in older adults (e.g., Falkenstein, Hoormann, \& Hohnsbein, 2001; Li, Hämmerer, Müller, Hommel, \& Lindenberger, 2009; Rabbitt, Osman, Moore, \& Stollery, 2001; Rush, Barch, \& Braver, 2006; Themanson, Hillman, \& Curtin, 2006; Williams, Ponesse, Schachar, Logan, \& Tannock, 1999).

The observed differences during child development and aging suggest that various processes that are relevant for processing response conflicts function less well at both ends of the lifespan. 
Developmental theories and recent empirical findings corroborate this view. Extant findings suggest that deficits in resisting interference and in using cue information for attentional control are likely to contribute to lifespan changes in dealing with response conflicts during tasks involving response suppression (Braver, Barch, \& Keys et al., 2001; Braver, Satpute, Rush, Racine, \& Barch, 2005; Burke \& Osborne, 2007; Dempster, 1992; Kane, Hasher, Stoltzfus, Zacks, \& Connelly, 1994; Lorsbach \& Reimer, 2008; Rush et al., 2006). More specifically, compared to younger adults, children and adolescents are less able to inhibit unwanted action tendencies, while older adults show greater difficulties in using contextual cues to aid the top-down attentional control of conflicting actions (Braver, Barch, \& Gray et al., 2001; Braver, Barch, \& Keys et al., 2001; Braver et al., 2005; Burke \& Osborne, 2007; Houdé, 1995; Pascual-Leone, 1983; Rush et al., 2006; see Craik \& Bialystok, 2006; Diamond, 2006; Geier \& Luna, 2009 for reviews).

Hence, cue utilization and response suppression appear to contribute differently to response-conflict processing during maturation and aging. When investigating the development of response-conflict processing, it is thus helpful to examine (a) both child development and aging, and (b) changes in other taskrelevant cognitive functions in order to better characterize the multifaceted nature of response-conflict processing. Therefore, in addition to ERPs assumed to be related to conflict monitoring, in the present study we also investigate ERP correlates of the individuals' ability to (a) use cue information in guiding actions, and (b) anticipate or suppress responses. In the following, we review the ERPs thought to reflect the cognitive functions in question. We will start with ERPs reflecting conflict monitoring (Nogo-N2) and response suppression (Nogo-P3), before describing ERPs reflecting the use of cue information (Cue-P3) and response anticipation (Cue-CNV).

\subsection{ERP correlates of response conflict monitoring, cue \\ utilization, response suppression, and response anticipation}

In a typical response suppression task, such as the stop-signal task or the continuous performance task (CPT), participants are asked to suppress a prepotent response (Lappin \& Eriksen, 1966; Rosvold, Mirsky, Sarason, Bransome, \& Beck, 1956). To facilitate the investigation of inhibitory mechanisms, a bias for response execution is often established by introducing response deadlines or by increasing the probability of Go trials, that is, trials in which a response needs to be executed (e.g., Jonkman, 2006). ERPs to Go and Nogo stimuli typically show a negative deflection in a time window 200-300 ms following the imperative stimulus, and a prominent positive deflection in an interval of $300-500 \mathrm{~ms}$ post stimulus, referred to as N2 and $\mathrm{P} 3$, respectively. The N2 is maximal over the frontal regions and larger after Nogo (Nogo-N2) than after Go stimuli (Go-N2). Previous findings suggest that the Nogo-N2 arises from the anterior cingulate cortex or medial frontal generators (Bekker, Kenemans, \& Verbaten, 2005; Nieuwenhuis et al., 2003), which are known to be involved in response conflict monitoring (Ridderinkhof et al., 2004). The P3 after Nogo stimuli (Nogo-P3) is also larger than the P3 after Go stimuli (Go-P3). The Nogo-P3 shows a central scalp distribution, whereas the Go-P3 is maximal in amplitude at parietal electrodes (Bruin \& Wijers, 2002; Eimer, 1993; Karlin, Martz, \& Mordkoff, 1970; Kok, 1986; Pfefferbaum, Ford, Weller, \& Kopell, 1985). The generator of the Nogo-P3 has been localized in inferior frontal cortices (Bokura, Yamaguchi, \& Kobayashi, 2001).

The Nogo-N2 was originally assumed to reflect an inhibitory mechanism (e.g., Jodo \& Kayama, 1992; Nakata et al., 2006; van Boxtel, van der Molen, Jennings, \& Brunia, 2001). However, more recent views see the Nogo-N2 as a signal emitted from a more general conflict monitoring system, which is activated by tasks that include several conflicting response tendencies such as the flanker task (Nieuwenhuis et al., 2003). Strong evidence in favor of this view comes from studies showing that the N2 is actually larger for Go trials than for Nogo trials if the less frequent response type is a Go response. This finding supports the view that (a) the response tendencies for either executing or suppressing a response are in conflict during tasks involving response suppression; (b) the conflict is larger for the less frequent or less prepotent response type, resulting in a larger N2 amplitude (Donkers \& van Boxtel, 2004; Nieuwenhuis et al., 2003). In contrast, the Nogo-P3 has been more consistently linked to response suppression (Bekker, Kenemans, \& Verbaten, 2004; Bruin \& Wijers, 2002; Jonkman, 2006; Karlin et al., 1970; Schupp, Lutzenberger, Rau, \& Birbaumer, 1994; Smith, Johnstone, \& Barry, 2008). This distinction of the processes reflected in the Nogo-N2 and the Nogo-P3 is supported by evidence showing that when participants are asked to only count Go and Nogo stimuli, the Nogo-N2 is still present while the Nogo-P3 is reduced (Bruin \& Wijers, 2002; Donkers, Nieuwenhuis, \& van Boxtel, 2005)

With respect to assessing the ability to utilize cue information, the ERP responses of participants to cues that are instructive in terms of preparing their responses can be examined. For example, in the AX-CPT task, participants are asked to respond to the letter $\mathrm{X}$ only if this letter is preceded by the letter A (e.g., Braver, Barch, \& Gray et al., 2001; Braver, Barch, \& Keys et al., 2001). A larger P3 to cue stimuli has been interpreted as indicating a higher amount of information extracted from the cue (Gratton et al., 1990; Wright, Geffen, \& Geffen, 1993). In addition to the utilization of cue information, the investigation of EEG activity prior to the target stimulus offers a means of assessing indices of response anticipation. Here, the late contingent negative variation (CNV), seems to be a promising candidate, as it has been linked to central processes of motor preparation or stimulus expectation (e.g., Damen \& Brunia, 1994; Gomez, Marco, \& Grau, 2003; Rektor et al., 2004; Ulrich, Leuthold, \& Sommer, 1998). The putamen, pre SMA, inferior parietal cortex, and primary motor cortex have been suggested to contribute to the generation of the CNV (Gomez et al., 2003; Rektor et al., 2004).

In summary, assessing ERPs during a cued CPT provides the possibility for examining three task-relevant cognitive functions in addition to the process of conflict monitoring: (a) the use of cue information (Cue-P3), (b) ERPs reflecting motor control (response suppression as evident in the Nogo-P3), and (c) response anticipation as evident in the $\mathrm{CNV}$.

\subsection{Lifespan differences in ERP correlates of response conflict} monitoring, cue utilization, response suppression, and response anticipation

To date, ERP studies on developmental differences in inhibition and conflict monitoring are scarce. Existing studies that have compared children and adolescents in an age range spanning from 6 years to young adulthood report a decrease of the Nogo-N2 with increasing age (Johnstone et al., 2007; Johnstone, Pleffer, Barry, Clarke, \& Smith, 2005; Jonkman, 2006; Jonkman et al., 2003). This suggests a reduction of monitored conflict or responsiveness of the monitoring system with maturation. A recent study by Lamm, Zelazo, and Lewis (2006) showed that the decrease in NogoN2 amplitude is not a mere artifact of peripheral changes with chronological age (e.g., increasing skull thickness with increasing age) but related to the increase in the executive functions with increasing age. To the best of our knowledge, thus far only two studies have investigated age differences in the Nogo-N2 from early to late adulthood. In these studies, smaller N2 amplitudes have been observed in older adults (Czigler, Csibra, \& Ambro, 1996; Falkenstein, Hoormann, \& Hohnsbein, 2002).

The amplitude of the Nogo-P3 has been shown to increase during childhood and adolescence (Johnstone et al., 2007, 2005; Jonkman, 2006). The difference in amplitude between the Nogo-P3 
and the Go-P3 seems to increase as well, indicating improvements in the ability to suppress unwanted responses. So far, only a handful of studies have focused on senescent changes in the Go- and Nogo-P3. Generally, the amplitude of the P3 component has been found to decrease with advancing adult age (Czigler et al., 1996; Falkenstein et al., 2002; Pfefferbaum \& Ford, 1988). In addition, the results of the few studies conducted so far suggest that the difference between Nogo-P3 and Go-P3 amplitudes observed in younger adults is smaller in old age. Note, however, that the older adults in the study by Falkenstein et al. (2002) were relatively young (mean age $=58.3$ years), and that the ERPs reported by Pfefferbaum and Ford (1988) were based on three midline electrodes only. Thus, the currently available evidence may not present a typical or complete picture of adult age differences in ERP correlates of response conflict monitoring.

To date, only a few studies have used EEG to assess ERP components that are related to cue processing (e.g. Cue-P3) in addition to other known ERP components associated with conflict monitoring and response suppression (Jonkman, 2006; Jonkman et al., 2003). Jonkman $(2003,2006)$ demonstrated that children showed a larger Cue-P3 than adults. This was interpreted as reflecting a stronger cue-driven Go-stimulus expectation in children. The CNV was shown to be reduced in children relative to young adults or absent until the age of 11 or 12 years (Jonkman, 2006; Jonkman et al., 2003; Segalowitz \& Davies, 2004). This finding has been interpreted as indicating weaker response anticipation in children. Similar observations have been made for older adults (Botzel, Mayer, Oertel, \& Paulus, 1995).

\subsection{Study aim and hypotheses}

The aim of this study was to arrive at a more complete picture of lifespan differences in the various processes relevant for handling response conflicts, with a particular focus on ERP correlates of conflict monitoring, cue utilization, response anticipation and response suppression. Given that ERP studies on response conflict monitoring have, thus far, either focused on child development or aging, the present study aims at directly comparing ERP correlates of conflict monitoring, cue utilization, response anticipation and response suppression in a lifespan sample covering middle childhood to old age.

In light of developmental theories and empirical findings suggesting that children and adolescents are less able to inhibit unwanted action tendencies and thus are more prone to errors during response conflicts (e.g., Davies et al., 2004; Houdé, 1995; Jonkman, 2006; Pascual-Leone, 1983; see Craik \& Bialystok, 2006; Diamond, 2006; Geier \& Luna, 2009 for reviews), we expected smaller amplitudes of the ERPs reflecting motor control in children and adolescents (Nogo-P3 and Cue-CNV; cf: Jonkman et al., 2003; Jonkman, 2006) relative to young adults. Regarding age differences in adulthood, behavioral as well as imaging studies show that older adults have greater difficulties in using cue information to guide attentional control while processing response conflicts than younger adults (Braver, Barch, Keys et al., 2001; Braver et al., 2005; Burke \& Osborne, 2007; Rush et al., 2006). We thus expected reduced amplitudes of ERPs reflecting cue processing (Cue-P3) and conflict monitoring (Nogo-N2) in the elderly (cf. Czigler et al., 1996).

\section{Method}

\subsection{Participants}

The sample included 182 participants covering four age groups: 45 children ( 22 girls), 44 adolescents ( 22 girls), 46 younger adults ( 22 women), and 47 older adults ( 22 women). Given that response conflict monitoring evolves more rapidly from childhood to early adulthood than from early to late adulthood (e.g., Li et al., 2004), the age ranges within age groups were smaller in the groups of children and adolescents than in the groups of younger and older adults (see Table 1). Data from one adolescent and one younger adult who did not comply with the task instructions were excluded from the analyses. Furthermore, one adolescent subject was excluded due to technical problems during EEG recordings.

The educational level of the participants was comparatively high. The majority of the children were still attending elementary school (81\%), with some already attending the Gymnasium, the college preparatory track of high school. Most of the adolescents were attending the Gymnasium (78\%), the majority of the younger adults were enrolled at a university (91\%), and most of the older adults held academic high school diploma (53\%) or vocational school diploma (34\%). All subjects were right-handed (Oldfield Questionnaire: LQ> 80; Oldfield, 1971).

In a separate test session prior to the EEG session, the Digit Symbol Substitution test (DSS; Wechsler, 1981) and a modified version of the Spot-a-Word test (Lehrl, 1977; see also Lindenberger, Mayr, \& Kliegl, 1993) were administered as marker tests of perceptual speed and verbal knowledge, respectively (Horn, 1989). According to two-component theories of life span cognition (Baltes, 1987; Lindenberger, 2001), fluid intelligence relies relatively more on basic cognitive mechanics, whereas crystallized intelligence depends relatively more on experience and acquired knowledge. Thus, it can be expected that the lifespan trajectories of marker tests of fluid intelligence match more closely the maturation- and senescence-related age trajectories of brain development than the lifespan trajectories of measures of crystallized intelligence. As can be seen in Table 1, in our sample, DSS performance increased from childhood to early adulthood and decreased from early to late adulthood (planned contrast: $t=11.66, p<.01, d=1.81, r=.67$ ), whereas performance on the Spot-a-Word test increased with age $\left(X^{2}(3, N=182)=129.5, p<.01\right)$. The observed dissociation between lifespan age gradients of these two tests in our sample is consistent with well-established empirical evidence on the development of these two facets of intelligence obtained in larger and more representative samples (e.g., Li et al., 2004). Informed consent was obtained from each participant or parent of the participant before testing. The study was approved by the Ethics Committee of the Max Planck Institute for Human Development. Participants were paid $10 €$ for the first, and $7 €$ for every following hour of the experiment.

\subsection{Procedure}

Participants were comfortably seated in an electrically and acoustically shielded room. The distance to the computer screen was $80 \mathrm{~cm}$. Each session started with a relaxation phase of three

Table 1

Sample description: age, perceptual speed (digit symbol score), and verbal knowledge (spot-a-word) across the age groups.

\begin{tabular}{|c|c|c|c|}
\hline Age group & Mean age (years) & Mean digit symbol score (correctly filled fields) & Mean spot-a-word score (correct responses) \\
\hline Children & $10.15(0.59)$ & $35.58(7.67)$ & $4.64(4.28)$ \\
\hline Adolescents & $14.42(0.55)$ & $52.16(10.13)$ & $12.55(6.08)$ \\
\hline Younger adults & $24.27(2.07)$ & $62.24(12.07)$ & $21.35(5.63)$ \\
\hline Older adults & $71.24(2.91)$ & $45.55(8.66)$ & $26.43(5.16)$ \\
\hline
\end{tabular}

Note: S.D. in parentheses. 
minutes ( $1.5 \mathrm{~m}$ with eyes closed and $1.5 \mathrm{~m}$ with eyes open). Thereafter, subjects performed a modified version of the Continuous Performance Task (Rosvold et al., 1956). We modified a variant of the CPT task, the AX-CPT task (Braver, Barch, \& Gray et al., 2001) for testing with children. During the CPT task, 12 squares of different colors were presented one at a time in succession on a white screen. The blue square was the Cue stimulus and the yellow square was the Target stimulus. Participants were instructed to respond as fast as possible by pressing a button with their right index finger whenever the blue square was followed by the yellow square. The remaining 10 colored squares were Non-Cue stimuli and, like the Cue stimulus, were not to be responded to with a button press. There were altogether 270 Go pairs (Cue followed by Target) and 195 Nogo pairs, with 65 pairs for each possible type of Nogo pair: prime-based Nogo pair (Cue followed by Non-Target), responsebased Nogo pair (Non-Cue followed by Target), and Non-Cue Nogo pair (Non-Cue followed by Non-Target). Note that other studies that used the cued variant of the CPT task refered to these trial types as $\mathrm{AX}, \mathrm{AY}, \mathrm{BX}$ and BY trials, respectively.

Based on prior assessments of electrophysiological correlates during this task, only the prime-based Nogo trials were used for the Go-Nogo comparison in the EEG components (cf. Jonkman, 2006) since response conflict is assumed to be largest on this type of Nogo pair. The ratio of Go responses to Nogo responses over all trials was $58-42 \%$. The total number of trials was 930 trials, which were divided into 5 blocks of 186 trials each. Before the task started, participants underwent a practice block with a sequence of 20 stimuli. The complete task took approximately $35 \mathrm{~min}$ to complete. After each block, participants were allowed to take a short self-timed break and received feedback informing them how many correct and how many late responses (exceeding a response time limit, see below) they had made up to this point in time. They were also reminded to respond as quickly as possible.

\subsection{Stimulus material and trial structure}

Stimulus materials were chosen to be suitable for all age groups including children. The colored squares used in the task were $1.07^{\circ}$ tall and wide and presented in the center of the screen. At the beginning of each trial, a colored square was presented for $200 \mathrm{~ms}$ before being followed by a blank screen. A fixation cross $\left(0.35^{\circ}\right.$ tall and wide), presented after the blank screen, indicated the response time limit. It appeared $650 \mathrm{~ms}$ after the beginning of the trial for children and older adults and $500 \mathrm{~ms}$ after the beginning of the trial for adolescents and younger adults. These response time limits were determined in pilot experiments such that the four age groups would be motivated to the same extent to respond as quickly and as accurately as possible. The fixation cross remained on the screen for $1500 \mathrm{~ms}$ (in the case of children and older adults) or $1650 \mathrm{~ms}$ (in the case of adolescents and younger adults), resulting in a total trial duration of $2150 \mathrm{~ms}$.

\subsection{EEG recordings and analyses}

EEG was recorded continuously (BrainAmp DC amplifiers, Brain Products $\mathrm{GmbH}$, Gilching, Germany) from $64 \mathrm{Ag} / \mathrm{AgCl}$ electrodes placed according to the 10-10 system in an elastic cap (Braincap, BrainVision), using BrainVision Recorder. The sampling rate was $1000 \mathrm{~Hz}$ with a bandpass filter applied in the range of $0.01-250 \mathrm{~Hz}$. EEG recordings were referenced online to the right mastoid. The ground was positioned above the forehead. Impedances were kept below $5 \mathrm{k} \Omega$. Vertical and horizontal electro-oculograms were recorded next to each eye and below the left eye.

Using BrainVision Analyzer, the recorded data were rereferenced to a linked mastoid reference. Using the Fieldtrip software package (for more details see http://www.ru.nl/fcdonders/ fieldtrip), the data were segmented into epochs of $1.5 \mathrm{~s}$ before and $2.5 \mathrm{~s}$ after the onset of the colored square. Epochs or channels with severe muscular artifacts or saturated recordings were excluded manually. An average of $12.01 \%$ of the trials had to be removed from the EEG data; children: $19.25 \%$, adolescents: $12.56 \%$, younger adults: $8.41 \%$, older adults: $8.10 \%$. The number of trials per condition was included as a covariate in the multivariate repeated measures analyses of variance (MANOVA) on the condition and age effects at the electrodes with maximal effects (see statistical analyses below). All main and interaction effects reported below proved to be robust with respect to differences in the number of trials per condition. The prepared data were subjected to an ICA decomposition using EEGLAB (Delorme \& Makeig, 2004). ICA components of ocular and muscular artifacts were removed from the data. The recombined data were bandpass-filtered in the range of $0.5-25 \mathrm{~Hz}$ and epoched according to the time windows of interest $(1000 \mathrm{~ms}$ after stimulus onset for the Go and Nogo comparison and $2150 \mathrm{~ms}$ after stimulus onset for the Cue and Non-Cue stimulus comparison). Baseline corrections were applied on the epoched data with respect to a $100 \mathrm{~ms}$ prestimulus baseline. Epochs for the analyses of the CNV were not bandpass-filtered, but only lowpass-filtered at $25 \mathrm{~Hz}$. ERPs were obtained by averaging across trials for each electrode and condition for each subject, and across subjects within each age group.

Latencies and amplitudes of the P2, N2 and P3 components following Go stimuli (Target after a Cue) and Nogo stimuli (primebased Nogo: Non-Target after a Cue) were defined as the most positive (or negative) peaks in the individual averages in the time windows $100-250 \mathrm{~ms}, 200-350 \mathrm{~ms}$ and $200-500 \mathrm{~ms}$, respectively. For the P3 component following Non-Cue and Cue stimuli, the most positive peak in the time windows $300-600 \mathrm{~ms}$ after stimulus onset was chosen. The CNV amplitude was determined by identifying the amplitude immediately preceding the onset of the next stimulus, that is, the measurement of the CNV occurred at $2150 \mathrm{~ms}$ (i.e., the total trial duration) following the onset of the Cue or Non-Cue stimuli (cf. Falkenstein et al., 2002; Johnstone et al., 2007; Jonkman, 2006 for comparable time windows in developmental studies).

\subsection{Exclusion criteria for behavioral data}

Reaction times on Go trials were prepared prior to data analyses by excluding reaction times faster than $100 \mathrm{~ms}$ and slower than 2000 ms. Then, for each age group, reaction times deviating more than 3.5 standard deviations from the mean of the age group were excluded. Altogether, $5.6 \%$ of the reaction times needed to be excluded in children, $4.0 \%$ in adolescents, $2.3 \%$ in younger adults, and $1.3 \%$ in the older adults.

\subsection{Statistical analyses}

Data were analyzed using SPSS (Release 15.0.0, Sept 6th 2006) and SAS (SAS 9.1.3, Windows Version 5.2.3790). Deviations from normality were corrected by transforming the data (square root and logarithmic transformations). In the case of unequal variances, tests that allow for unequal variances like the SAS PROC MIXED procedure assuming unstructured variances were used. Non-parametric tests were used when transformations failed to establish normality of the data in all four age groups.

To identify differences in scalp distribution between the age groups, multivariate repeated measures analyses of variance were performed on normalized data (cf. scaling method following McCarthy \& Wood, 1985) for each ERP component on 9 leads (F3, Fz, F4, C3, Cz, C4, P3, Pz, P4). Condition (Go vs Nogo or Cue vs Non-Cue), laterality (3 levels: medium-left, mid-sagittal, mediumright), and the anterior-posterior distinction (3 levels: frontal, central, parietal) were within-subject factors, whereas age group 
Table 2

Mean of median reaction time and percent errors across the age groups.

\begin{tabular}{|c|c|c|c|c|}
\hline Age group & Go RT (ms) & Misses (\%) & Prime-based errors (\%) & Response-based errors (\%) \\
\hline Children & $292(39)$ & $9.7(7.6)$ & $42.1(18.2)$ & $13.8(12.2)$ \\
\hline Adolescents & $267(30)$ & $8.2(8.1)$ & $27.6(15.4)$ & $7.1(6.1)$ \\
\hline Younger adults & $275(29)$ & $3.7(4.5)$ & $14.3(11.9)$ & $4.6(4.6)$ \\
\hline Older adults & $321(41)$ & $3.4(4.5)$ & $13.1(10.4)$ & $5.6(4.3)$ \\
\hline
\end{tabular}

Note: S.D. in parentheses.

(children, adolescents, younger adults, and older adults) was the between-subject factor. Given that we were most interested in testing age differences in scalp distributions, we focused on interactions including the factor of age group. Furthermore, only reliable effects with effect sizes $r_{I}>.35$ were considered for follow-up analyses to avoid reporting non-essential effects.

To assess lifespan differences in Go and Nogo, Cue and NonCue conditions, the ERP responses from predetermined electrodes based on existing developmental literature were compared across the four age groups using multivariate repeated measures analyses of variance as outlined above. The N2 following Go and Nogo stimuli was examined at electrode Fz (e.g., Jonkman, 2006), the Go-P3 at electrode $\mathrm{Pz}$ and the Nogo-P3 at electrode $\mathrm{Cz}$ (e.g., Czigler et al., 1996). Cue and Non-Cue P3 were investigated at electrode Pz (cf. Jonkman, 2006), with the exception of the age group of the older adults, where the maximal effect after Non-Cue stimuli appeared at electrode $\mathrm{Cz}$ (see below for details). Finally, the CNV following Cue and Non-Cue stimuli was assessed at electrode Cz (cf. Jonkman, 2006).

Significant main effects of age group were followed by three planned contrasts (which did not assume equal variances) to further characterize differences between age groups. The first contrast tested for a linear pattern across the age groups, the second for a curvilinear pattern across the age groups and the third for a difference between the younger (children and adolescents) and the adult (younger and older adults) age groups. Furthermore, Games-Howell pairwise comparisons, which correct for type I error accumulation and are suitable for samples with unequal variances, were calculated to investigate differences between the age groups. Significant interactions of age and condition were analyzed further using paired samples $t$-tests to assess differences between the levels of the condition factor separately for each age group. The intraclass correlation coefficient $r_{I}$ was calculated as the effect size indicator for MANOVA, and Pearson's $r$ was computed as an effect size for correlational analyses. Cohen's $d$ was calculated as the effect size indicator for planned contrasts and pairwise comparisons across the age groups; the effect sizes of the latter where based on pooled standard deviations to account for differences in variances between age groups. To allow for further comparisons of the effect sizes across statistical tests, we report in addition the transformation of Cohen's $d$ to Pearson's $r$ (cf. Fern \& Monroe, 1996). Behavioral data were compared across age groups using univariate analyses of variance robust to unequal variances across the age groups, as well as planned contrasts and pairwise corrected comparisons as described above for the analyses of the EEG data.

\section{Results}

\subsection{Behavioral performance: reaction times and accuracy}

The behavioral data are summarized in Table 2 . The median reaction times on Go responses differed significantly across the four age groups, $F(3,178)=21.00, p<.01, r_{I}=.51$. Planned contrasts confirmed the expected curvilinear pattern across the age groups, with children and older adults having the largest median reaction times, $t=6.80, p<.01, d=1.06, r=0.47$. A comparison of the median reac- tion times in children and older adults revealed that older adults had significantly longer reaction times than children, mean difference $28.59 \mathrm{~ms}, t=3.43, p<.01, d=.72, r=.39$.

Prime-based errors were the most common type of errors, followed by response-based errors and misses, main effect of error type: $X^{2}(2,172)=156, p<.01$, indicating that suppressing a prepotent response tendency was indeed the critical event in the CPT task. When the four age groups were compared separately for each type of error, significant differences in error frequency across the lifespan were observed for prime-based errors, $F(3,168)=16.31, p<.01$, $r_{I}=.47$, response-based errors, $F(3,153)=8.42, p<.01, r_{I}=.38$, and misses, $F(3,177)=38.99, p<.01, r_{I}=.63$. Planned contrasts between age groups performed separately for each error type revealed that children and adolescents committed more errors than younger and older adults for prime-based errors, $t=10.06, p<.01, d=1.5$, $r=0.60$, response-based errors, $t=3.99, p<.01, d=.65, r=.31$, and misses: $t=6.78, p<.01, d=1.03, r=.46$. As expected, children and adolescents had greater difficulties in suppressing inappropriate responses than younger and older adults.

Pairwise comparisons that were conducted to follow up on the contrast effects further examined the patterns across the lifespan for the different types of errors. The frequency of prime-based errors decreased from childhood to early adulthood and did not differ between younger and older adults (mean differences: children-adolescents $14.5 \%, p<.01, d=.85$, $r=.39$; adolescents - younger adults $13.3 \%, p<.01, d=1.01, r=.45$; younger-older adults $1.2 \%, p=1.0$ ). The frequency of responsebased errors was higher in children than in any of the other age groups (mean differences: children-adolescents $6.7 \%$, $p=.036, d=.56, r=.27$; adolescents - younger adults $2.6 \%, p=.59$; younger - older adults $1.0 \%, p=1.0$. Finally, misses were more frequent in children and adolescents than in younger and older adults (mean differences: children-adolescents $1.5 \%, p=.49$; adolescents - younger adults $4.5 \%, p<.01, d=.78, r=.36$; younger - older adults $0.3 \%, p=1.0$.).

\subsection{Speed-accuracy trade-offs}

In all four age groups, we observed strong negative correlations between the median reaction time and the frequency of prime-based errors, indicative of a speed-accuracy trade-off: children: $r=-.70, p<.01$; adolescents: $r=-.74, r<.01$; younger adults: $r=-.63, p<.01$; older adults: $r=-.63, p<.01$. To examine whether a speed-accuracy trade-off was also present within individuals, we binned individual reaction time data for every 30 trials, and split up the bins into those with low and high median reaction times for each particular individual. The number of prime-based errors committed within a 30-trial bin was then averaged separately for the bins with large median reaction times and the bins with small median reaction times. A multivariate repeated measures analysis with the factors age group and number of errors (levels: errors during slower responding, errors during faster responding) revealed that participants committed more prime-based errors during periods of faster responding, $F(1,179)=25.57, p<.01, r_{I}=.35$, demonstrating that the speed-accuracy trade-off was also present at the intraindividual level. Importantly, the age group $\times$ number 

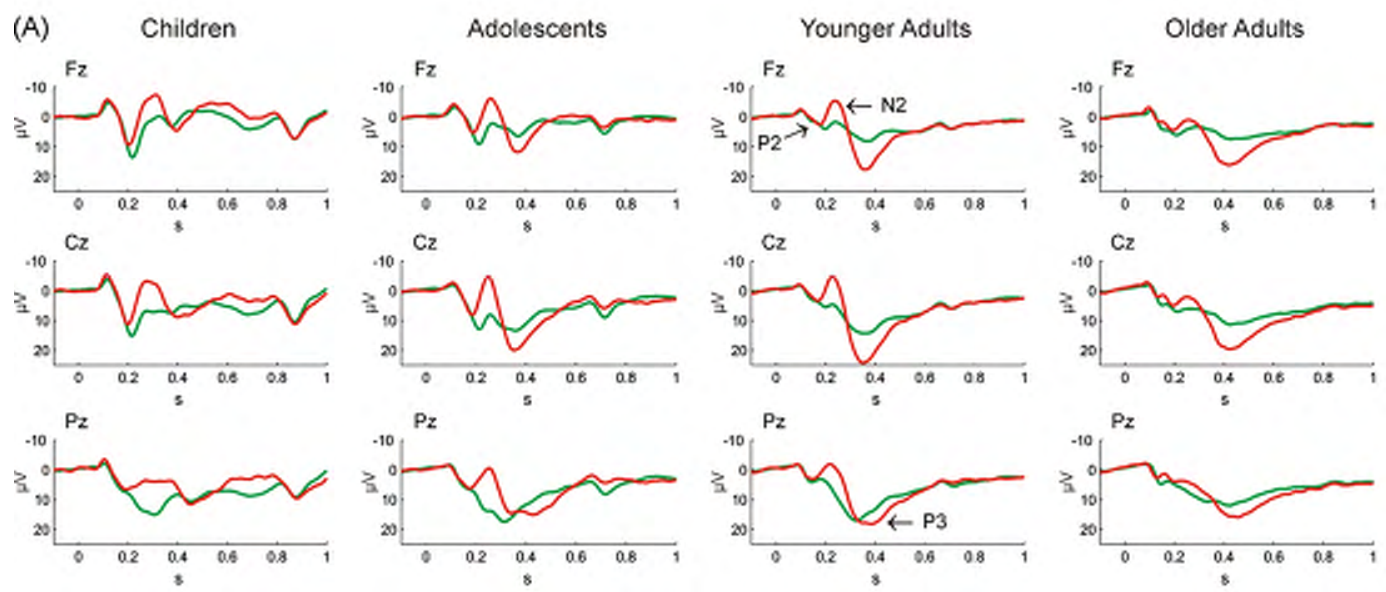

(B)
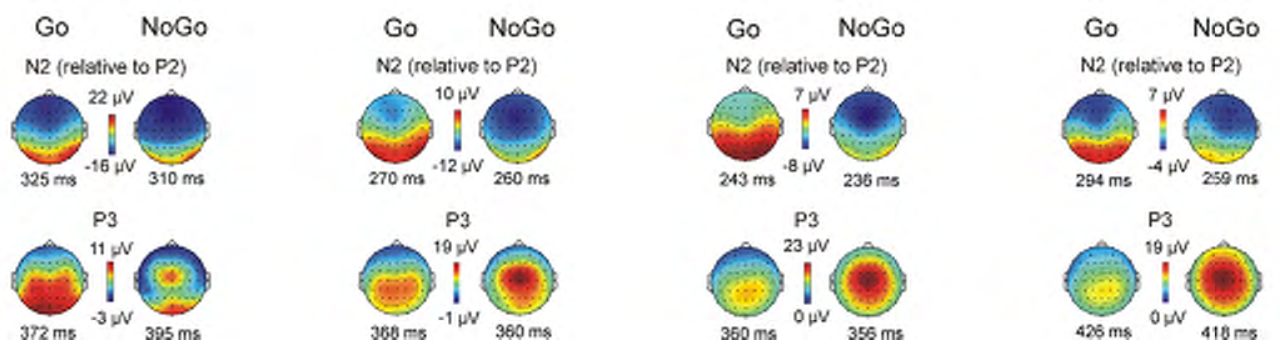

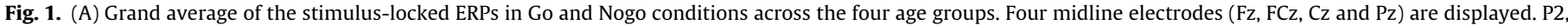

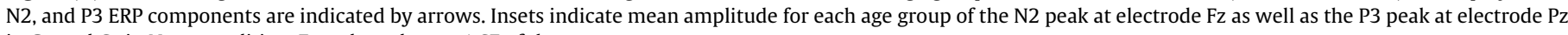
in $\mathrm{Go}$ and $\mathrm{Cz}$ in Nogo condition. Error bars denote $1 \mathrm{SE}$ of the mean.

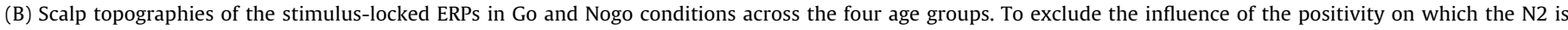

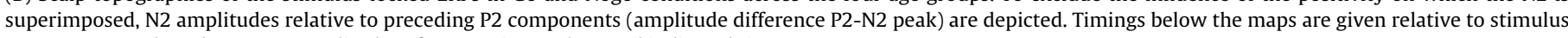
onset. Maps are based on mean amplitudes of a $50 \mathrm{~ms}$ interval around indicated time.

of errors interaction was not statistically significant, $F(3,179)=.61$, $p=.61$, indicating that the average magnitude of the intraindividual speed-accuracy trade-off did not differ reliably across the four age groups. Taken together, these results indicate that speed-accuracy trade-offs did not differentially affect variations in error proneness between and within participants in each of the four age groups. Thus, age-group differences in speed-accuracy trade-off are unlikely to confound the observed lifespan trends.

\subsection{Age differences in the topography of the ERPs}

As expected, the P3 amplitude following Go stimuli was largest at $\mathrm{Pz}$ while the $\mathrm{P} 3$ following Nogo stimuli was maximal at $\mathrm{Cz}\left(F(2.00,27.00)=13.36, p<.01, r_{I}=.71\right.$; cf. Czigler et al., 1996). The P3 following Go and Nogo stimuli showed an age group $\times$ anterior-posterior interaction, $F(4.00,237.33)=9.33$, $p<.01, r_{I}=.37$. Follow up analyses revealed a comparatively stronger effect at $\mathrm{Pz}$ in children (cf. Fig. 1) that was apparent especially following Nogo stimuli (mean difference $\mathrm{Cz}-\mathrm{Pz}$, children: $0.09 \mu \mathrm{V}, p=0.16$; adolescents: $0.31 \mu \mathrm{V}, p<.01, d=2.56$, $r=.79$; younger adults: $0.36 \mu \mathrm{V}, p<.01, d=3.39, r=.86$; older adults: $0.33 \mu \mathrm{V}, p<.01, d=2.74, r=.81$ ). In contrast, older adults showed a comparatively reduced increase from $\mathrm{Cz}$ to $\mathrm{Pz}$ (cf. Fig. 1) in the P3 following Go stimuli (mean difference $\mathrm{Cz}-\mathrm{Pz}$, children: $-0.30 \mu \mathrm{V}, p<0.1, d=2.32, r=.76$; adolescents: $-0.13 \mu \mathrm{V}, p<.01$, $d=0.94, r=.42$; younger adults: $-0.13 \mu \mathrm{V}, p<.01, d=1.04, r=.46$; older adults: $-0.02 \mu \mathrm{V}, p=.65$ ). The more posteriorly localized P3 in children especially during Nogo stimuli might indicate the need for a recruitment of additional posterior sources (cf. Jonkman, Sniedt, \& Kemner, 2007 for a similar finding with respect to the N2 following Nogo stimuli in children). The more anterior Go P3 in older adults is in line with evidence of a frontal shift of P3s with aging (i.e., P3b,
P3a, Go P3, and Nogo P3; see Fallgatter, Mueller, \& Strick, 1999; Fjell \& Walhovd, 2004), which has been proposed to be related to volume decreases in medial prefrontal areas and volume increases in orbitofrontal cortex in the elderly (Fjell, Walhovd, \& Reinvang, 2005). Since the topographical differences in Go and Nogo P3 in children and older adults were not reflected in a reliably larger amplitude at electrode sites other than those suggested by the literature (cf. Czigler et al., 1996), we assessed Go and Nogo-P3 at the standard electrode sites (i.e., Nogo-P3 at electrode Cz and Go P3 at electrode Pz) for comparisons within Go or Nogo conditions. However, comparisons across conditions were performed across both, $\mathrm{Cz}$ and $\mathrm{Pz}$, taking into account the observed topographical differences of the Go and Nogo condition.

The P3 following Cue and Non-Cue stimuli showed a reliable age group $\times$ anterior-posterior interaction $(F(3.97,235.33)=13.05$, $p<.01, r_{I}=.42$ ) indicating age-related differences in the topological distribution of the P3. An additional threeway age group $\times$ condition $\times$ anterior-posterior interaction $\left(F(3.86,228.88)=3.67, p<.01, r_{I}=.24\right)$ revealed that these agerelated differences in the topological distribution were different for Cue and Non-Cue stimuli. Post hoc tests showed that the P3 in older adults was larger at electrode $\mathrm{Cz}$ following Non-Cue stimuli (mean difference $\mathrm{Cz}-\mathrm{Pz}$ after Non-Cue stimuli, children: $-0.33 \mu \mathrm{V}, p<0.1, d=2.17, r=.74$; adolescents: $-0.36 \mu \mathrm{V}, p<.01$, $d=1.86, r=.68$; younger adults: $-0.23 \mu \mathrm{V}, p<.01, d=1.31, r=.55$; older adults: $0.10 \mu \mathrm{V}, p=.12$ ). As can be seen in the scalp maps in Fig. 2, older adults showed a centrally distributed P3 following Non-Cue stimuli, while the other age groups show P3s with a parietal maximum. This suggests that the processes captured by the P3 following Non-Cue stimuli may differ across age groups. We thus examined differences between the age groups on Non-Cue stimuli at electrode $\mathrm{Cz}$ in addition to electrode $\mathrm{Pz}$. Also, condition 

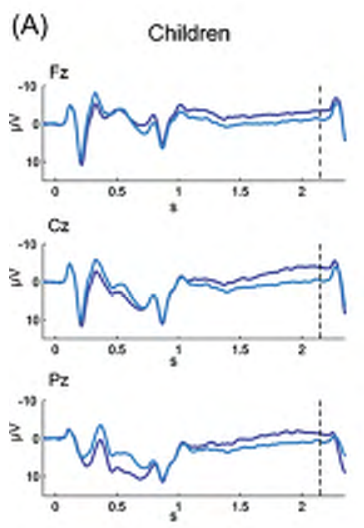

(B)

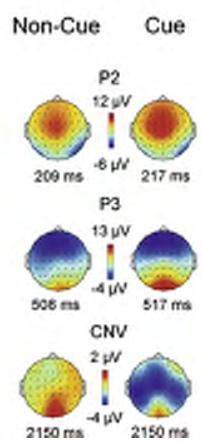

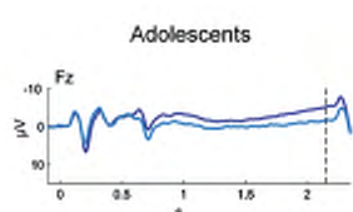
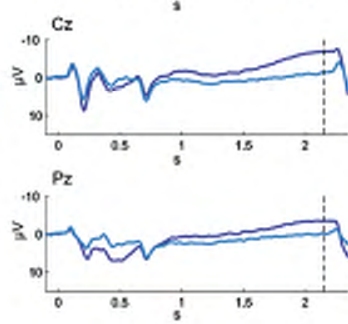

Non-Cue Cue

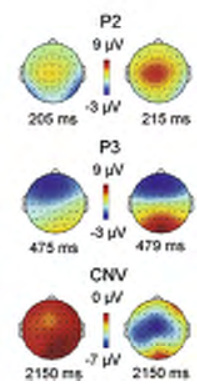

Younger Adults
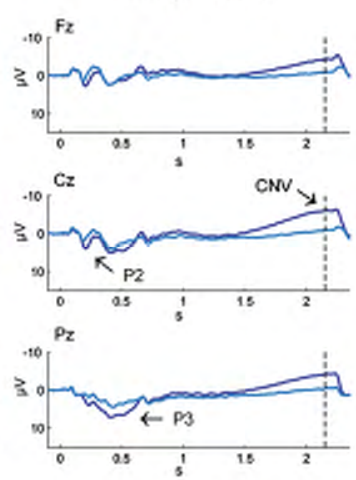

Non-Cue Cue

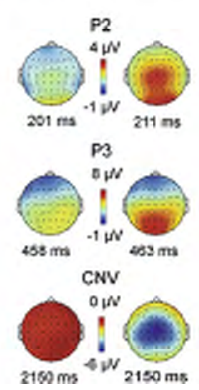

Older Adults
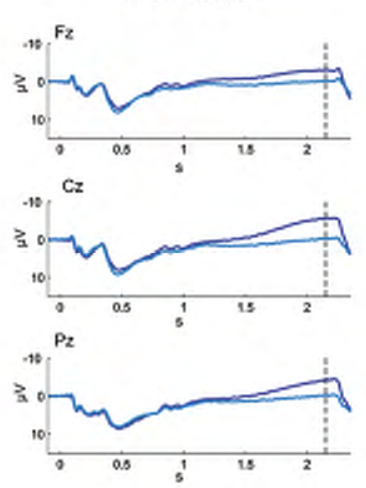

Non-Cue Cue
Cue Non-Cue
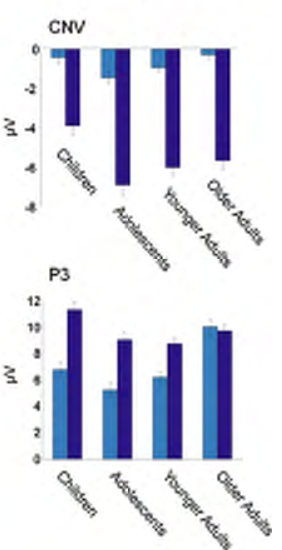

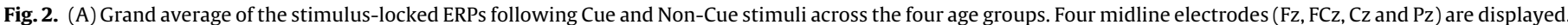

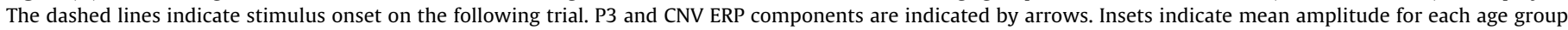

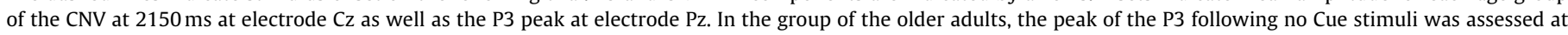
electrode $\mathrm{Cz}$ (see Section 3). Error bars denote $1 \mathrm{SE}$ of the mean.

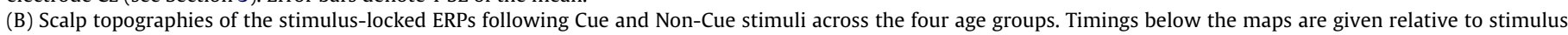
onset. Maps are based on mean amplitudes of a $50 \mathrm{~ms}$ interval around indicated time.

differences between Cue and Non-Cue-P3s were examined at both, electrodes $\mathrm{Cz}$ and $\mathrm{Pz}$.

\subsection{Age differences in conflict monitoring as reflected in the Go-N2 vs. Nogo-N2 effect}

To assess age differences in the size of the monitoring reaction as evident in the N2 amplitude, peak amplitudes at electrode Fz were compared across the four age groups on Go and Nogo trials (see Fig. 1). As expected, the amplitude of the N2 was larger on Nogo than on Go trials, $F(1,134)=342.18, p<.01, r_{I}=.85$, suggesting higher levels of response conflict on trials requiring response suppression. Furthermore, N2 amplitudes differed significantly between age groups, $F(3,81.2)=23.95, p<.01, r_{I}=.69$. Planned contrasts between age groups, performed separately for Go and Nogo trials, revealed a linear decrease of $\mathrm{N} 2$ amplitude with increasing age in the Go condition, $t=3.78, p<.01, d=0.68, r=.32$, and the Nogo condition, $t=8.76, p<.01, d=1.65, r=.64$. As revealed by the significant condition $\mathrm{x}$ age group interaction, the linear amplitude decrease with age group was steeper in the Nogo than in the Go condition, $F(3,86)=24.63, p<.01, r_{I}=.68$. This might be related to age differences in the amount of monitored conflict in the Nogo condition or in the responsiveness of the monitoring system to conflicts.

\subsection{Age differences in response suppression as reflected in the Go-P3 vs. Nogo-P3 effect}

In line with previous findings, the Go P3 amplitude was largest at electrode Pz while the P3 following Nogo stimuli was largest at electrode Cz (Czigler et al., 1996; see topographical analyses above and Fig. 1). When examined at electrodes $\mathrm{Cz}$ and Pz together, the
P3 following Nogo trials was larger than the P3 following Go trials, $F(1,174)=64.03, p<.01, r_{I}=.51$. Furthermore, P3 amplitudes differed between age groups, $F(3,174)=12.25, p<.01, r_{I}=.42$. Planned contrasts, performed separately for Go (at electrode Pz) and Nogo trials (at electrode $\mathrm{Cz}$ ), revealed a curvilinear lifespan pattern, with children and older adults having the smallest P3 amplitudes, GoP3: $t=3.76, p<.01, d=.58, r=.28$; Nogo-P3: $t=6.14, p<.01, d=.96$, $r=.43$. In addition, on Nogo trials we found a reliable linear trend, $t=5.70, p<.01, d=.96, r=.43$, due to the comparatively small NogoP3 amplitudes in children (see Fig. 1). This effect was confirmed in a reliable condition $\mathrm{x}$ age group interaction, $F(3,174)=12.18, p<.01$, $r_{I}=.42$.

\subsection{Age differences in cue utilization as reflected in the Non-Cue-P3 vs. Cue-P3 effect}

Tested across electrodes $\mathrm{Cz}$ and $\mathrm{Pz}$, the amplitude of the CueP3 was larger following a Cue than following a Non-Cue stimulus, $F(1,178.0)=89.19, p<.01, r_{I}=.58$ (cf. Fig. 2 ). Furthermore, the amplitudes of Cue-P3 and Non-Cue-P3 showed a reliable interaction between age group and cue type $\left(F(3,178.0)=19.89, p<.01, r_{I}=.50\right)$. Follow-up analyses revealed that the P3 amplitude at electrode $\mathrm{Pz}$ following Cue stimuli was largest in children. Mean differences between age groups for the $\mathrm{P} 3$ (at Pz) following Cue stimuli were as follows: children - adolescents $2.27 \mu \mathrm{V}, p=.03, d=.54, r=.26$; adolescents - younger adults $0.31 \mu \mathrm{V}, p=1.0$; younger - older adults $-0.99 \mu \mathrm{V}, p=1.0$. In the case of the older adults, as compared to younger adults, reliably larger P3 amplitudes after Non-Cue stimuli but not after Cue stimuli (see above) were observed (mean differences for Non-Cue-P3 at electrode Pz: younger - older adults $-3.29 \mu \mathrm{V}, p<.01, d=1.07, r=.47$, at electrode $\mathrm{Cz}$ : younger - older 
adults $-5.01 \mu \mathrm{V}, p<.01, d=1.34, r=.55)$. As will be discussed below, together with the different scalp distribution on the Non-Cue-P3 in older adults (only the older adults have a centrally distributed P3 following Non-Cue stimuli, see topographical analyses above), these analyses suggest that the processes captured by the P3 following Non-Cue stimuli may differ in the older adults.

\subsection{Age differences in response anticipation as reflected in the CNV-Cue vs. CNV-Non-Cue effect}

As can be seen in Fig. 2, the CNV was larger after a Cue than after a Non-Cue stimulus, $F(1,149)=524.76, p<.01, r_{I}=.88$. Hence, as expected, participants were showing greater response anticipation after Cue stimuli that signal a Go response. The CNV amplitude differed between age groups, $F(3,81.2)=5.74, p<.01, r_{I}=.42$, especially following a Cue stimulus, $F(3,74.4)=4.54, p<.01, r_{I}=.39$. To follow-up on this interaction effect, we conducted pairwise corrected comparisons between the age groups for the CNV following Cue stimuli. CNV amplitudes following Cue stimuli were significantly smaller in children than in the other age groups; mean differences: children - adolescents $-3.01 \mu \mathrm{V}, p<.01, d=.95, r=.43$; adolescents - younger adults $.88 \mu \mathrm{V}, p=.57$, younger - older adults $.36 \mu \mathrm{V}, p=.93$. In line with earlier findings (Jonkman, 2006; Jonkman et al., 2003; Segalowitz \& Davies, 2004), children showed smaller CNVs in response to action-relevant cues.

\subsection{Correlations among the ERP components}

The amplitude of the CNV after Cue stimuli and Nogo-P3 amplitudes were negatively correlated in adolescents $(r=-.48, p<.01)$, younger adults $(r=-.55, p<.01)$, and older adults $(r=-.49, p<.01)$, indicating that participants with a larger CNV also showed a larger Nogo-P3 (see Fig. 3). However, this was not the case among the children age group, $r=-.17, p=.28$. The correlations among adolescents, younger adults, and older adults remained reliable even after controlling for DSS performance and age differences within the age groups. The Nogo-N2 was not reliably correlated to the CNV amplitude following Cue stimuli ( $p$ values ranged from .80 to .41 ).

\section{Discussion}

In this study, we used a cued CPT to identify and compare ERP components of cue utilization, response anticipation, and response suppression and response conflict monitoring among children, adolescents, younger adults, and older adults. In the following, we first summarize the behavioral and the ERP findings, and then discuss the major implications of our findings.

The behavioral data revealed clear age group differences. Median reaction times were longer for children and older adults than for adolescents and younger adults, confirming the curvilinear lifespan pattern in speed of responding (cf. Li et al., 2004). It is worth noting that children, who responded under the same response deadline than older adults, answered considerably faster than older adults. The most frequent type of error in all age groups was the prime-based error, indicating that the inhibition of a prepared response is indeed the critical event in a speeded CPT. Prime-based errors were more frequent in children and adolescents than in younger and older adults, confirming the claim that the ability to suppress prepared responses matures relatively late (cf. Diamond, 2006; Geier \& Luna, 2009). In addition, misses and response-based errors also occurred more frequently in children and adolescents than in younger and older adults. These two types of errors are supposed to result from inattentiveness or insufficient use of cue information (Braver, Barch, \& Gray et al., 2001; Braver, Barch, \& Keys et al., 2001; Halperin et al., 1988). It is noteworthy that adolescents committed less prime-based errors than children, while children and adolescents did not differ in the frequency of misses. The greater reduction in prime-based errors than in misses from childhood to adolescence supports the view that the ability to suppress prepared responses, as indexed by prime-based errors, shows particularly pronounced changes from middle childhood to adolescence. In line with this observation, the amplitude of the Nogo-P3 component, which is assumed to reflect response suppression, was larger in adolescents than in children. Complementing our finding, Jonkman (2006) reported more frequent misses in younger children aged 6 or 7 years as compared to 9 or 10 year olds, but a comparable number of prime-based errors, and suggested that the ability to control impulsive behavior matures later than attentional processes. Finally, we found that the response accuracies of younger adults and older adults did not differ reliably. Although response deadlines as well as reaction times were longer in the groups of the older adults, speed-accuracy trade-off appeared to be comparable in the two adult age groups, suggesting that the comparable error rate is not related to a more conservative response strategy among older adults. Furthermore, this finding is in line with a previous study using a CPT with short delay without different response deadlines for younger and older adults (Braver, Barch, \& Gray et al., 2001; Braver, Barch, \& Keys et al., 2001).

\subsection{Lifespan differences in Cue utilization and response conflict monitoring}

We assessed electrophysiological correlates of CPT performance to delineate the contributions of cue utilization, response anticipation, and response suppression to lifespan differences in response conflict monitoring. For this purpose, EEG signals were locked to the cue or to the target, respectively. In agreement with earlier findings (for a review, see Müller, Brehmer, von Oertzen, Li, \& Lindenberger, 2008), the amplitude of the N2 after Go stimuli decreased with increasing age (see Fig. 1). The Nogo-N2 proved to be larger than the Go-N2 in all age groups, suggesting that all age groups experienced more conflict in Nogo than in Go trials. However, the conflictrelated amplitude difference between Nogo and Go trials decreased from childhood to early adulthood, and was drastically diminished in older adults relative to the other three age groups.

The observed reduction in Nogo-N2 amplitude from childhood to early adulthood suggests a decrease of experienced conflict during this age period (Johnstone et al., 2007; Jonkman, 2006) and has been related to an increase in executive functions (Lamm et al., 2006). This view is supported by the linear decrease in the frequency of prime-based errors, which suggests an increasing ability to manage response conflicts, probably reflecting the late maturation of relevant brain areas such as the ACC and the prefrontal cortices (Raz et al., 2005; Resnick, Pham, Kraut, Zonderman, \& Davatzikos, 2003). As we will argue in more detail below, the further reduction of the Nogo-N2 among older adults may require a different explanation.

So far, only few studies (e.g., Bekker et al., 2004; Jonkman, 2006; van Leeuwen et al., 1998) have investigated how components reflecting attention to cue stimuli might be related to indices of response conflict, such as the Nogo-N2. In these studies, age differences during maturation in Nogo-N2 amplitude were linked to age differences in cue processing. Specifically, a stronger reaction to the cue in children was interpreted as reflecting a stronger Go expectation on the following trial. This stronger Go expectation would then result in a stronger deception of an expectation when a Non-target follows the cue, similar to the stronger monitoring signal to unexpected or infrequent outcomes during reinforcement learning tasks (Gehring \& Willoughby, 2002; Holroyd, Larsen, \& Cohen, 2004). Given that the N2 is also found with tasks that do not require overt responding (Bruin, Wijers, \& van Staveren, 2001; Burle, Vidal, \& Bonnet, 2004; Smith et al., 2008), it seems plausible 
that the expectation of a critical target stimulus might contribute to the conflict signal as reflected in the Nogo-N2 in children.

In our study, ERPs reflecting the amount of information extracted from the Cue stimulus (Cue-P3) were larger in children and adolescents (see Fig. 2). Our findings hence confirm the decrease of the Cue-P3 amplitude from childhood to adulthood (Jonkman, 2006), and suggest a stronger Go expectancy in children and adolescents than in younger and older adults. Furthermore, the parallel age-associated reductions in the Nogo-N2 and the Cue-P3 towards adulthood support the assumption that expecting an upcoming target stimulus contributes to the response conflict reflected in the Nogo-N2 during maturation. However, it should be kept in mind that this view of potentially different functions underlying performance monitoring in children and adults is based on age-group comparisons of the different profiles of ERP components that are related to monitoring and motor control processes. A more thorough test of this proposition needs to investigate the responsiveness of the performance monitoring system across the lifespan by systematically manipulating the information provided by external feedback and internal motor-control related monitoring demands (e.g., by a block-wise manipulation of cue-target contingencies and different levels of conflicting response mappings).

Comparing the P3 amplitudes after Non-Cue stimuli to those after Cue stimuli across age groups suggested that older adults differed in their reaction to Non-Cue stimuli from the other age groups. In children, adolescents, and younger adults, P3 components were larger after a Cue stimulus than after a Non-Cue stimulus, suggesting that participants in these age groups devoted more attention to Cue stimuli, which informed them about a probable response on the following trial. They paid less attention to Non-Cue stimuli, which informed them that no response needed to be prepared. In contrast, older adults showed a P3a component after Non-Cue stimuli. The P3a is thought to reflect involuntary and transient allocation of attention to novel or unexpected stimuli (Fjell \& Walhovd, 2004; Squires, Squires, \& Hillyard, 1975; Wetzel \& Schröger, 2007). Allocation of attention to irrelevant or unexpected stimuli comes with costs of attentional distraction such as prolonged reaction times and decreased hit rates (e.g., Roeber, Berti, \& Schröger, 2003). Also, a larger P3a seems to be related to greater attentional distraction costs; when participants receive information about the forthcoming deviant, they exhibit a reduced P3a and showed less reaction time prolongation (Sussman, Winkler, \& Schröger, 2003). We do not wish to claim that a non-Cue stimulus in the CPT is noninformative, as it indicates that no response needs to be prepared for the following trial, even if it is a target stimulus. Nevertheless, at least in the present study, this type of stimulus is less frequent than Cue and Target stimuli and also more variable in its appearance (e.g., color). In contrast to the P3a found in older adults, with largest amplitudes at central electrodes, children, adolescents, and younger adults showed a P3 with parietal maxima in reaction to both Cue and Non-Cue stimuli. Taken together, these differences suggest that older adults were extracting less taskrelevant information from cues than individuals from the other three age groups. Instead, older adults may have processed the Non-Cue stimuli as being deviant from the Cue or Target stimuli and hence may have failed to use them effectively for suppressing a response on the next trial. Recently, Vallesi, Stuss, McIntosh, and Picton (2009) reported a similar result in a non-cued GoNogo task. As in the present study, a central P3a component to irrelevant and less frequent Nogo stimuli was found in older adults only. The notion that older adults are more distractible and less likely to prepare responses is consistent with studies reporting greater response time costs for switching between different task sets with advancing adult age (e.g., Kray, Li, \& Lindenberger, 2002; Kray \& Lindenberger, 2000; Mayr, 2001).
We thus provide electrophysiological evidence that the observed senescent declines in the Nogo-N2 during response conflict monitoring may reflect difficulties in maintaining attentional focus. This interpretation also fits the larger picture that agingrelated impairments in cognitive control affect the capacity for generating or maintaining action goals (Braver, Barch, \& Gray et al., 2001; Braver, Barch, \& Keys et al., 2001; Braver et al., 2005; De Jong, 2001; De Jong, Berendsen, \& Cools, 1999; cf. Craik, Routh, \& Broadbent, 1983) since they suggest that already the acquisition of the information needed to form this goal is compromised in older adults. For instance, Braver and colleagues (2001) proposed a cognitive model that attributes the observed deficits in maintaining context information during aging to a faster decay and a reduced reliability of context representation due to the senescent decline in prefrontal dopaminergic modulation.

Alternatively, given evidence that heightened activity in the ACC might be related to action selection rather than to conflict monitoring (Roelofs, van Turennout, \& Coles, 2006), the lower Nogo-N2 in the older adults might point to a weaker action selection in this age group. According to this alternative explanation, the pattern of lower Nogo-N2s and Non-Cue P3a components in older adults would suggest that older adults experience greater difficulties in attentional and action-related selection.

In the context of a comparable speed-accuracy trade-off, older adults did not differ in error frequencies from younger adults, even though the Nogo-N2 amplitude was much lower than among younger adults. Although a reduced N2 amplitude in older adults is a common finding on similar conflict monitoring tasks such as e.g. the Flanker task (e.g. Falkenstein et al., 2001), the lower N2 in older adults in the presence of a comparable number of errors raises the question whether the assumed relationship between the amount of conflict and the proneness to errors is the same in younger and older adults. However, as outlined above, a reduced attentional focus to Cue and Non-Cue stimuli among older adults may result in reduced representations of cue information and hence a reduced build-up of response tendencies according to the conflict monitoring model (cf. Botvinick et al., 2001; Yeung et al., 2004). Weaker response tendencies would then cause less conflict, as apparent in a lower Nogo-N2 component, while not necessarily harming response accuracy as long as both correct and incorrect response tendencies are reduced to the same degree (Yeung \& Cohen, 2006). The same line of reasoning may also help to explain why response times were disproportionately prolonged (also when compared to children who were responding under the same response deadline) among older adults, as activations would need more time to reach a given threshold (Yeung $\&$ Cohen, 2006).

\subsection{Lifespan differences in response anticipation and response suppression}

In line with previous findings (Polich, 1997; Falkenstein et al., 2002), the Go-P3 amplitude decreased from early to late adulthood (see Fig. 1). Again, the ERP component that is of interest in the investigation of lifespan age differences in coping with response conflicts is the component following Nogo trials, where a prepared response must be suppressed. Adolescents, younger adults, and older adults showed a marked increase in P3 amplitude after Nogo stimuli, but children showed no increase of the P3 amplitude in the Nogo as compared to the Go trials. Apparently, children were less able to invest cognitive effort into the suppression of an inappropriate response (cf. Diamond, 2006).

The late CNV is thought to reflect central processes of motor preparation or stimulus expectation (e.g., Damen \& Brunia, 1994; Ulrich et al., 1998). In agreement with this interpretation, the amplitude of the CNV was larger after a Cue than after a Non-Cue stimulus (see Fig. 2). Furthermore, paralleling the age differences 

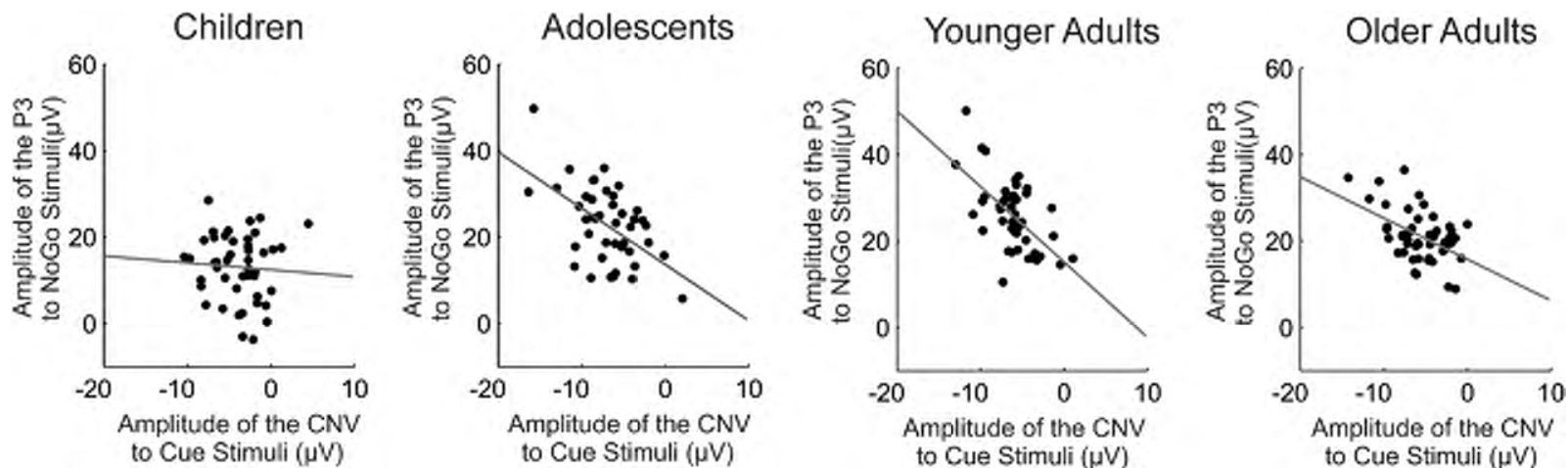

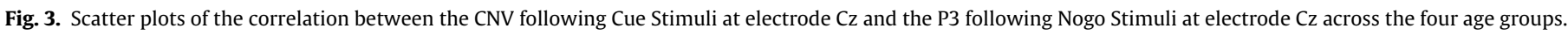

for the Nogo-P3, the CNV following a Cue was smallest in children when compared to the other age groups. We also found that adolescents, younger adults, and older adults with a larger late CNV following Cue stimuli also exhibited a larger Nogo-P3 (for a previous report on this relationship in younger adults, see Smith, Johnstone, \& Barry, 2006). The observed correlations in the three age groups were robust against controlling for the size of the CNV following Non-Cue stimuli and the size of the Go-P3, indicating that they were not an epiphenomenon of individual differences in CNV or P3 amplitudes in general. Rather, this correlation appears to reflect interindividual differences in the intensity of motor control processes (Fig. 3).

In contrast, the correlation between the CNV following Cue stimuli and Nogo-P3 amplitudes was not reliable among children. We conclude that children not only show reduced levels of response anticipation and response suppression but that these preparatory processes are also less tightly coupled. This pattern of findings suggests that the motor control system is not yet fully functional in children, presumably pointing to a late maturation of the frontoparietal motor control network (Garavan, Ross, \& Stein, 1999; Jonkman, 2006; Luna \& Sweeney, 2004; Watanabe et al., 2002). This interpretation is corroborated by the observed age differences in commission errors, which were by far the most frequent among children. At the same time, and somewhat ironically, children also showed the strongest ERP responses to cues. In middle childhood, then, the behavioral risks of one's ability to use cues for guiding actions are not yet brought under control by an equally developed ability to suppress action when needed. This pattern might be seen as corroborating the assumption of a transition from exogenous to endogenous control of behavior during cognitive maturation (Luna \& Sweeney, 2004).

\section{Outlook}

In this study, we were able to show that different components related to response conflict monitoring follow dissociable lifespan trajectories. Relative to younger adults, children showed less response suppression, whereas older adults showed greater attentional distractibility and less efficient conflict monitoring. The combined assessment of cue utilization, response anticipation and response suppression was helpful in improving the understanding of the lifespan changes in the relationship of conflict monitoring processes to other task-relevant cognitive functions. Future studies need to further probe the dependency of conflict monitoring processes on other task-relevant cognitive functions by systematically manipulating the relevance of cue information and closely examining the contribution of lifespan differences in the fronto-parietal motor control network.

\section{Acknowledgements}

This study was supported by the German Research Foundation's grant to Shu-Chen Li and Ulman Lindenberger for a subproject (Li 515/8-1) in the research group on Conflicts As Signals (DFG FOR 778). We thank our student research assistants and interns Katja Breitenbach, Beate Czerwon, Minh Tam Luong, Carlos Picchioni, Viola Störmer, Natalie Trumpp, Katja Zschenderlein, and other research assistants for their valuable support during data collection. Parts of this article were written while UL was a fellow at the Center for Advanced Study in the Behavioral Sciences at Stanford University. We thank Bernd Wischnewski, Markus Bauer and Markus Werkle-Bergner for consultations on EEG recordings and data analyses. We also thank Julius Verrel, Sascha Schroeder and the 'Neuromodulation of Lifespan Cognition' team for fruitful discussions.

\section{References}

Baltes, P. B. (1987). Theoretical propositions of life-span developmental psychology: On the dynamics between growth and decline. Developmental Psychology, 23, 611-626.

Bekker, E. M., Kenemans, J. L., \& Verbaten, M. N. (2004). Electrophysiological correlates of attention, inhibition, sensitivity and bias in a continuous performance task. Clinical Neurophysiology, 115, 2001-2013.

Bekker, E. M., Kenemans, J. L., \& Verbaten, M. N. (2005). Source analysis of the N2 in a cued Go/NoGo task. Cognitive Brain Research, 22, 221-231.

Bokura, H., Yamaguchi, S., \& Kobayashi, S. (2001). Electrophysiological correlates for response inhibition in a Go/NoGo task. Clinical Neurophysiology, 112, 22242232.

Botvinick, M. M., Braver, T. S., Barch, D. M., Carter, C. S., \& Cohen, J. D. (2001). Conflict monitoring and cognitive control. Psychological Review, 108, 624-652.

Botvinick, M. M., Cohen, J. D., \& Carter, C. S. (2004). Conflict monitoring and anterior cingulate cortex: An update. Trends in Cognitive Sciences, 8, 539-546.

Botzel, K., Mayer, M., Oertel, W. H., \& Paulus, W. (1995). Frontal and parietal premovement slow brain potentials in Parkinson's disease and aging. Movement Disorders, 10, 85-91.

Braver, T. S., Barch, D. M., Gray, J. R., Molfese, D. L., \& Snyder, A. (2001). Anterior cingulate cortex and response conflict: Effects of frequency, inhibition and errors. Cerebral Cortex, 11, 825-836.

Braver, T. S., Barch, D. M., Keys, B. A., Carter, C. S., Cohen, J. D., Kaye, J. A., et al. (2001) Context processing in older adults: Evidence for a theory relating cognitive control to neurobiology in healthy aging. Journal of Experimental Psychology: General, $130,746-763$.

Braver, T. S., Satpute, A. B., Rush, B. K., Racine, C. A., \& Barch, D. M. (2005). Context processing and context maintenance in healthy aging and early stage dementia of the Alzheimer's type. Psychology and Aging, 20, 33-46.

Bruin, K. J., \& Wijers, A. A. (2002). Inhibition, response mode, and stimulus probability: A comparative event-related potential study. Clinical Neurophysiology, 113, 1172-1182.

Bruin, K. J., Wijers, A. A., \& van Staveren, A. S. (2001). Response priming in a go/nogo task: Do we have to explain the go/nogo N2 effect in terms of response activation instead of inhibition? Clinical Neurophysiology, 112, 1660-1671.

Burke, D. M., \& Osborne, G. (2007). Aging and inhibition deficits: Where are the effects? In D. S. Gorfein \& C. M. MacLeod (Eds.), Inhibition in cognition (pp. 163-183). Washington, DC: American Psychological Association.

Burle, B., Vidal, F., \& Bonnet, M. (2004). Electroencephalographic nogo potentials in a no-movement context: The case of motor imagery in humans. Neuroscience Letters, 360, 77-80. 
Craik, F. I. M., \& Bialystok, E. (2006). Cognition through the lifespan: Mechanisms of change. Trends in Cognitive Sciences, 10, 131-138.

Craik, F. I. M., Routh, D. A., \& Broadbent, D. E. (1983). On the transfer of information from temporary to permanent memory. Philosophical Transactions of the Royal Society of London B Biological Sciences, 302, 341-359.

Czigler, I., Csibra, G., \& Ambro, A. (1996). Aging, stimulus identification and the effect of probability: An event-related potential study. Biological Psychology, 43(1), 27-40.

Damen, E. J., \& Brunia, C. H. (1994). Is a stimulus conveying task-relevant information a sufficient condition to elicit a stimulus-preceding negativity? Psychophysiology, 31, 129-139.

Davies, P. L., Segalowitz, S. J., \& Gavin, W. J. (2004). Development of responsemonitoring ERPs in 7- to 25-year-olds. Developmental Neuropsychology, 25, 355-376.

De Jong, R. (2001). Adult age differences in goal activation and goal maintenance. European Journal of Cognitive Psychology, 13, 71-89.

De Jong, R., Berendsen, E., \& Cools, R. (1999). Goal neglect and inhibitory limitations: Dissociable causes of interference effects in conflict situations. Acta Psychologica, $101,379-394$.

Delorme, A., \& Makeig, S. (2004). EEGLAB: An open source toolbox for analysis of single-trial EEG dynamics including independent component analysis. Journal of Neuroscience Methods, 134, 9-21.

Dempster, F. N. (1992). The rise and fall of the inhibitory mechanism: Toward a unified theory of cognitive development and aging. Developmental Review, 12, 45-75.

Diamond, A. (2006). The early development of executive functions. In E. Bialystok \& F. I. Craik (Eds.), Lifespan cognition: Mechanisms of change (pp. 70-95). New York: Oxford University Press.

Donkers, F. C., Nieuwenhuis, S., \& van Boxtel, G. J. (2005). Mediofrontal negativities in the absence of responding. Cognitive Brain Research, 25, 777-787.

Donkers, F. C., \& van Boxtel, G. J. (2004). The N2 in go/no-go tasks reflects conflict monitoring not response inhibition. Brain and Cognition, 56, 165-176.

Eimer, M. (1993). Effects of attention and stimulus probability on ERPs in a Go/NoGo task. Biological Psychology, 35, 123-138.

Falkenstein, M., Hoormann, J., \& Hohnsbein, J. (2001). Changes of error-related ERPs with age. Experimental Brain Research, 138, 258-262.

Falkenstein, M., Hoormann, J., \& Hohnsbein, J. (2002). Inhibition-related ERP components: Variation with modality, age, and time-on-task. Journal of Psychophysiology, 16, 167-175.

Fallgatter, A. J., Mueller, T. J., \& Strick, W. K. (1999). Age-related changes in the brain electrical correlates of response control. Clinical Neurophysiology, 110, 833-838.

Fern, E. F., \& Monroe, K. B. (1996). Effect-size estimates: Issues and problems in interpretation. The Journal of Consumer Research, 23, 89-105.

Fjell, A. M., \& Walhovd, K. B. (2004). Life-span changes in P3a. Psychophysiology, 41, 575-583.

Fjell, A. M., Walhovd, K. B., \& Reinvang, I. (2005). Age-dependent changes in distribution of P3a/P3b amplitude and thickness of the cerebral cortex. Neuroreport, 16(13), 1451-1454.

Garavan, H., Ross, T. J., \& Stein, E. A. (1999). Right hemispheric dominance of inhibitory control: An event-related functional MRI study. Proceedings of the National Academy of Sciences U S A, 96, 8301-8306.

Gehring, W. J., \& Willoughby, A. R. (2002). The medial frontal cortex and the rapid processing of monetary gains and losses. Science, 295, 2279-2282.

Geier, C., \& Luna, B. (2009). The maturation of incentive processing and cognitive control. Pharmacology, Biochemistry and Behavior, 93, 212-221.

Gomez, C. M., Marco, J., \& Grau, C. (2003). Preparatory visuo-motor cortical network of the contingent negative variation estimated by current density. Neuroimage, 20, 216-224.

Gratton, G., Bosco, C. M., Kramer, A. F., Coles, M. G., Wickens, C. D., \& Donchin, E. (1990). Event-related brain potentials as indices of information extraction and response priming. Electroencephalography and Clinical Neurophysiology, 75, 419-432.

Halperin, J. M., Wolf, L. E., Pascualvaca, D. M., Newcorn, J. H., Healey, J. M., O’Brien, J. D., et al. (1988). Differential assessment of attention and impulsivity in children. Journal of the American Academy of Child \& Adolescent Psychiatry, 27, 326329.

Holroyd, C. B., Larsen, J. T., \& Cohen, J. D. (2004). Context dependence of the event-related brain potential associated with reward and punishment. Psychophysiology, 41, 245-253.

Horn, J. L. (1989). Models of intelligence. In R. L. Linn (Ed.), Intelligence: Measurement, theory, and public policy (pp. 29-73). Urbana, IL: University of Illinois Press.

Houdé, O. (1995). Rationalité, développement. et inhibition. Paris: Universitaires de France.

Jodo, E., \& Kayama, Y. (1992). Relation of a negative ERP component to response inhibition in a Go/No-go task. Electroencephalography and Clinical Neurophysiology, $82,477-482$.

Johnstone, S. J., Dimoska, A., Smith, J. L., Barry, R. J., Pleffer, C. B., Chiswick, D., et al. (2007). The development of stop-signal and Go/Nogo response inhibition in children aged 7-12 years: Performance and event-related potential indices. International Journal of Psychophysiology, 63, 25-38.

Johnstone, S. J., Pleffer, C. B., Barry, R. J., Clarke, A. R., \& Smith, J. L. (2005). Development of inhibitory processing during the Go/Nogo task. Journal of Psychophysiology, 19, $11-23$.

Jonkman, L. M. (2006). The development of preparation, conflict monitoring and inhibition from early childhood to young adulthood: A Go/Nogo ERP study. Brain Research, 1097, 181-193.
Jonkman, L. M., Lansbergen, M., \& Stauder, J. E. (2003). Developmental differences in behavioral and event-related brain responses associated with response preparation and inhibition in a go/nogo task. Psychophysiology, 40, 752-761.

Jonkman, L. M., Sniedt, F. L., \& Kemner, C. (2007). Source localization of the Nogo-N2: A developmental study. Clinical Neurophysiology, 118(5), 1069-1077.

Kane, M. J., Hasher, L., Stoltzfus, E. R., Zacks, R. T., \& Connelly, S. L. (1994). Inhibitory attentional mechanisms and aging. Psychology and Aging, 9, 103-112.

Karlin, L., Martz, M. J., \& Mordkoff, A. M. (1970). Motor performance and sensoryevoked potentials. Electroencephalography and Clinical Neurophysiology, 28, 307-313.

Kok, A. (1986). Effects of degradation of visual stimulation on components of the event-related potential (ERP) in Go/NoGo reaction tasks. Biological Psychology, 23, 21-38.

Kray, J., Li, K. Z. H., \& Lindenberger, U. (2002). Age-related changes in task-switching components: The role of task uncertainty. Brain and Cognition, 49, 363-381.

Kray, J., \& Lindenberger, U. (2000). Adult age differences in task switching. Psychology and Aging, 15, 126-147.

Lamm, C., Zelazo, P. D., \& Lewis, M. D. (2006). Neural correlates of cognitive control in childhood and adolescence: Disentangling the contributions of age and executive function. Neuropsychologia, 44, 2139-2148.

Lappin, J. S., \& Eriksen, C. W. (1966). Use of a delayed signal to stop a visual reactiontime response. Journal of Experimental Psychology, 72, 805-811.

Lehrl, S. (1977). Mehrfachwahl-Wortschatz-test B (MWT-B). Erlangen: Straube.

Li, S.-C., Hämmerer, D., Müller, V., Hommel, B., \& Lindenberger, U. (2009). Lifespan development of stimulus-response conflict cost: Similarities and differences between maturation and senescence. Psychological Research, 73, 777-785.

Li, S.-C., Lindenberger, U., Hommel, B., Aschersleben, G., Prinz, W., \& Baltes, P. B. (2004). Transformations in the couplings among intellectual abilities and constituent cognitive processes across the life span. Psychological Science, 15, $155-163$.

Lindenberger, U. (2001). Lifespan theories of cognitive development. In N. J. Smelser \& P. B. Baltes (Eds.), International encyclopedia of the social and behavioral sciences (Vol. 13, pp. 8848-8854). Amsterdam: Elsevier.

Lindenberger, U., Mayr, U., \& Kliegl, R. (1993). Speed and intelligence in old age. Psychology and Aging, 8, 207-220.

Lorsbach, T. C., \& Reimer, J. F. (2008). Context processing and cognitive control in children and young adults. Journal of Genetic Psychology, 169, 34-50.

Luna, B., \& Sweeney, J. A. (2004). The emergence of collaborative brain function: FMRI studies of the development of response inhibition. Annals of the New York Academy of Sciences, 1021, 296-309.

McCarthy, G., \& Wood, C. C. (1985). Scalp distributions of event-related potentials: An ambiguity associated with analysis of variance models. Electroencephalography and Clinical Neurophysiology, 62(3), 203-208.

Mayr, U. (2001). Age differences in the selection of mental sets: The role of inhibition, stimulus ambiguity, and response-set overlap. Psychology and Aging, 16, 96-109.

Müller, V., Brehmer, Y., von Oertzen, T., Li, S. C., \& Lindenberger, U. (2008). Electrophysiological correlates of selective attention: A lifespan comparison. BMC Neuroscience, $9,18$.

Nakata, H., Inui, K., Wasaka, T., Tamura, Y., Akatsuka, K., Kida, T., et al. (2006). Higher anticipated force required a stronger inhibitory process in go/nogo tasks. Clinical Neurophysiology, 117, 1669-1676.

Nieuwenhuis, S., Yeung, N., van den Wildenberg, W., \& Ridderinkhof, K. R. (2003). Electrophysiological correlates of anterior cingulate function in a $\mathrm{Go} / \mathrm{NoGo}$ task: Effects of response conflict and trial type frequency. Cognitive, Affective, E Behavioral Neuroscience, 3, 17-26.

Oldfield, R. C. (1971). The assessment and analysis of handedness: The Edinburgh inventory. Neuropsychologia, 9, 97-113.

Pascual-Leone, J. (1983). Growing into human maturity: Toward a metasubjective theory of adulthood stages. In P. B. Baltes \& O. G. J. Brim (Eds.), Life-span development and behavior (pp. 117-156). New York: Academic Press.

Pfefferbaum, A., \& Ford, J. M. (1988). ERPs to stimuli requiring response production and inhibition: Effects of age, probability and visual noise. Electroencephalography and Clinical Neurophysiology, 71, 55-63.

Pfefferbaum, A., Ford, J. M., Weller, B. J., \& Kopell, B. S. (1985). ERPs to response production and inhibition. Electroencephalography and Clinical Neurophysiology, $60,423-434$.

Polich, J. (1997). EEG and ERP assessment of normal aging. Electroencephalography and Clinical Neurophysiology, 104, 244-256.

Rabbitt, P., Osman, P., Moore, B., \& Stollery, B. (2001). There are stable individual differences in performance variability, both from moment to moment and from day to day. The Quarterly Journal of Experimental Psychology A: Human Experimental Psychology, 54A, 981-1003.

Raz, N., Lindenberger, U., Rodrigue, K. M., Kennedy, K. M., Head, D., Williamson, A., et al. (2005). Regional brain changes in aging healthy adults: General trends, individual differences and modifiers. Cerebral Cortex, 15, 1676-1689.

Rektor, I., Bares, M., Kanovsky, P., Brazdil, M., Klajblova, I., Streitova, H., et al. (2004). Cognitive potentials in the basal ganglia-frontocortical circuits. An intracerebral recording study. Experimental Brain Research, 158, 289-301.

Resnick, S. M., Pham, D. L., Kraut, M. A., Zonderman, A. B., \& Davatzikos, C. (2003). Longitudinal magnetic resonance imaging studies of older adults: A shrinking brain. Journal of Neuroscience, 23, 3295-3301.

Ridderinkhof, K. R., Ullsperger, M., Crone, E. A., \& Nieuwenhuis, S. (2004). The role of the medial frontal cortex in cognitive control. Science, 306, 443-447.

Roeber, U., Berti, S., \& Schröger, E. (2003). Auditory distraction with different presentation rates: An event-related potential and behavioral study. Clinical Neurophysiology, 114, 341-349. 
3316

D. Hämmerer et al. / Neuropsychologia 48 (2010) 3305-3316

Roelofs, A., van Turennout, M., \& Coles, M. G. (2006). Anterior cingulate cortex activity can be independent of response conflict in Stroop-like tasks. Proceedings of the National Academy of Sciences US A, 103, 13884-13889.

Rosvold, H. E., Mirsky, A. F., Sarason, I., Bransome, E. D., Jr., \& Beck, L. H. (1956). A continuous performance test of brain damage. Journal of Consulting E Clinical Psychology, 20, 343-350.

Rush, B. K., Burch, D. M., \& Braver, T. S. (2006). Accounting for cognitive aging: Context processing, inhibition or processing speed? Aging, Neuropsychology and Cognition, 13, 588-610.

Rushworth, M. F., Walton, M. E., Kennerley, S. W., \& Bannerman, D. M. (2004). Action sets and decisions in the medial frontal cortex. Trends in Cognitive Sciences, 8 , 410-417.

Schupp, H. T., Lutzenberger, W., Ru, H., \& Birbaumer, N. (1994). Positive shifts of event-related potentials: A state of cortical disfacilitation as reflected by the startle reflex probe. Electroencephalography and Clinical Neurophysiology, 90, 135-144.

Segalowitz, S. J., \& Davies, P. L. (2004). Charting the maturation of the frontal lobe: An electrophysiological strategy. Brain and Cognition, 55, 116-133.

Smith, J. L., Johnstone, S. J., \& Barry, R. J. (2006). Effects of pre-stimulus processing on subsequent events in a warned Go/NoGo paradigm: Response preparation, execution and inhibition. International Journal of Psychophysiology, 61, 121133.

Smith, J. L., Johnstone, S. J., \& Barry, R. J. (2008). Movement-related potentials in the Go/NoGo task: The P3 reflects both cognitive and motor inhibition. Clinical Neurophysiology, 119, 704-714.

Squires, N. K., Squires, K. C., \& Hillyard, S. A. (1975). Two varieties of long-latency positive waves evoked by unpredictable auditory stimuli in man. Electroencephalography and Clinical Neurophysiology, 38, 387-401.

Sussman, E., Winkler, I., \& Schröger, E. (2003). Top-down control over involuntary attention switching in the auditory modality. Psychonomic Bulletin E Review, 10 , 630-637.

Themanson, J. R., Hellman, C. H., \& Curtin, J. J. (2006). Age and physical activity influences on action monitoring during task switching. Neurobiology of Aging, 27, 1335-1345.
Ulrich, R., Leuthold, H., \& Sommer, W. (1998). Motor programming of response force and movement direction. Psychophysiology, 35, 721-728.

Vallesi, A., Stuns, T. D., McIntosh, A. R., \& Piston, T. W. (2009). Age-related differences in processing irrelevant information: Evidence from event-related potentials. Neuropsychologia, 47, 577-586.

van Boxtel, G. J., van der Molen, M. W., Jennings, J. R., \& Brunia, C. H. (2001). A psychophysiological analysis of inhibitory motor control in the stop-signal paradigm. Biological Psychology, 58, 229-262.

van Leeuwen, T. H., Steinhausen, H. C., Overtoom, C. C., Pascual-Marqui, R. D., van't Klooster, B., Rothenberger, A., et al. (1998). The continuous performance test revisited with neuroelectric mapping: Impaired orienting in children with battenton deficits. Behavioural Brain Research, 94, 97-110.

Watanabe, J., Sugiura, M., Seato, K., Sato, Y., Maeda, Y., Matsue, Y., et al. (2002). The human prefrontal and parietal association cortices are involved in NO-GO performances: An event-related fMRI study. Neuroimage, 17, 12071216.

Wechsler, D. (1981). Wechsler Adult Intelligence Scale-R. New York: Psychological Corporation.

Tetzel, N., \& Schröger, E. (2007). Cognitive control of involuntary attention and distraction in children and adolescents. Brain Research, 1155, 134146.

Williams, B. R., Ponesse, J. S., Schachar, R. J., Logan, G. D., \& Tannock, R. (1999). Levelopment of inhibitory control across the life span. Developmental Psychology, 35, 205-213.

Wright, M. J., Geffen, G. M., \& Geffen, L. B. (1993). Event-related potentials associated with covert orientation of visual attention in Parkinson's disease. Neuropsychologa, 31, 1283-1297.

Young, N., Botvinick, M. M., \& Cohen, J. D. (2004). The neural basis of error detection: Conflict monitoring and the error-related negativity. Psychological Review, 111, 931-959.

Young, N., \& Cohen, J. D. (2006). The impact of cognitive deficits on conflict monitoring. Predictable dissociation between the error-related negativity and N2. Psychological Science, 17, 164-171. 